\title{
Performance Evaluation of Block Type and Comb Type Channel Estimation for OFDM System under Various Modulation Techniques.
}

\author{
Vivek Kanwar ${ }^{1}$, Deepika Sharma ${ }^{2}$, Himani Thakur ${ }^{3}$ \\ ${ }^{1}$ (Department of Electronics and Communication, Shoolini University, India) \\ ${ }^{2}$ (Department of Electronics and Communication, Shoolini University, India) \\ ${ }^{3}$ (Department of Electronics and Communication, Shoolini University, India)
}

\begin{abstract}
With the rapid growth of digital communication in recent years, the need for high speed data transmission is increased. Moreover, future wireless systems are expected to support a wide range of services which includes video, data and voice. OFDM is a technique for achieving high data rates in mobile environment, due to its resistance to ISI, which is a common problems found in high speed data communication [1]. In OFDM, modulation may be differential or coherent. When using differential modulation there is no need for a channel estimate but its performance is inferior to the coherent system. Coherent modulation requires the channel estimation which gives better performance but with relatively more complex receiver structure. Pilot Symbol Assisted Modulation is used to achieve reliable channel estimates by transmitting pilots along with data symbols. In this paper, the channel estimation techniques for OFDM systems based on pilot arrangement are investigated. The channel estimation based on comb type pilot arrangement is studied through different algorithms for both estimating channel at pilot frequencies and interpolating the channel [4]. The estimation of channel at pilot frequencies is based on LS and LMS. In addition, the channel estimation based on block type pilot arrangement is performed by sending pilots at every sub-channel and using this estimation for a specific number of following symbols. I have compared the performances of all schemes by measuring bit error rate with 16QAM, QPSK, and BPSK as modulation schemes, and multipath Rayleigh fading.
\end{abstract}

Keywords: Binary Phase Shift Key (BPSK),Bit Error Rate (BER),Least square(LS), Minimum Mean square Error (MMSE), Least Mean Square (LMS) and Orthogonal Frequency Division Multiplexing (OFDM).

\section{INTRODUCTION}

A dynamic estimation of channel is necessary before the demodulation of OFDM signals since the radio channel is frequency selective and time-variant for wideband mobile communication systems [1]. The channel estimation can be performed by either inserting pilot tones into all of the subcarriers of OFDM symbols with a specific period or inserting pilot tones into each OFDM symbol [3]. The first one, block type pilot channel estimation, has been developed under the assumption of slow fading channel. Even with decision feedback equalizer, this assumes that the channel transfer function is not changing very rapidly. The estimation of the channel for this block-type pilot arrangement can be based on LS or MMSE. The MMSE estimate has been shown to give 0-5 dB gain in SNR for the same mean square error of channel estimation over LS estimate [6]. A low-rank approximation is applied to linear MMSE by using the frequency correlation of the channel to eliminate the major drawback of MMSE, which is complexity. The later, the comb-type pilot channel estimation has been introduced to satisfy the need for equalizing the significant changes even in one OFDM block. The comb-type pilot channel estimation consists of algorithms to estimate the channel at pilot frequencies and to interpolate the channel. The estimation of the channel at the pilot frequencies for comb-type based channel estimation can be based on LS, MMSE or LMS. MMSE has been shown to perform much better than LS [1]. The complexity of MMSE is reduced by deriving an optimal low-rank estimator with singular-value decomposition. The interpolation of the channel for comb-type based channel estimation can depend on linear interpolation, second order interpolation, low-pass interpolation, spline cubic interpolation, and time domain interpolation or DFT based interpolation [4].

\section{OFDM SYSTEM AND CHANNEL MODEL}

At the transmitter side, the binary information is first grouped and mapped into complex-valued symbols according to the modulation by different mapping scheme, such as BPSK, QPSK, 16QAM, and 64QAM. Then there is a serial to parallel conversion to prepare different data groups for different OFDM subcarrier [6]. The mapped signals are modulated into $\mathrm{N}$ orthogonal subcarrier by the IFFT. A cyclic prefix (CP) is then added to the multiplexed IFFT output. Finally, the obtained signal is converted to a time continuous 
analog signal before it is transmitted through the channel [8]. At the receiver side, an inverse operation is carried out and the information data is detected. OFDM is a modulation technique where multiple low data rate carriers are combined by a transmitter to form a composite high data rate transmission.



The binary information is first grouped and mapped according to the modulation in "signal mapper". After inserting pilots either to all sub-carriers with a specific period or uniformly between the information data sequence, IDFT block is used to transform the data sequence of length $N\{X(k)\}$ into time domain signal $\{x(n)\}$ with the following equation

$$
x(n)=\operatorname{IDFT}\{x(k)\}=\sum_{k=0}^{N-1} X(k) e^{\frac{j 2 \pi k n}{N}}
$$

where $\mathrm{N}$ is the DFT length. Following IDFT block, guard time, which is chosen to be larger than the expected delay spread, is inserted to prevent inter-symbol interference. This guard time includes the cyclically extended part of OFDM symbol in order to eliminate inter-carrier interference (ICI). The resultant OFDM symbol is given as follow

$$
x_{f}(n)=\left\{\begin{array}{cc}
x(N+n), & n=-N_{g}, \ldots-N_{g}+1 \\
x(n), & n=0,1, \ldots \ldots \ldots N-1
\end{array}\right.
$$

where $\mathrm{N}_{\mathrm{g}}$ is the length of the guard interval. After following D/A converter, this signal will be sent from the transmitter with the assumption of the baseband system model. The transmitted signal will pass through the frequency selective time varying fading channel with additive noise. The received signal is given by:

$$
\mathrm{y}_{\mathrm{f}}=\mathrm{x}_{\mathrm{f}}(\mathrm{n}) * \mathrm{H}(\mathrm{n})+\mathrm{w}(\mathrm{n})
$$

Where $\mathrm{w}(\mathrm{n})$ is additive white Gaussian noise and $\mathrm{h}(\mathrm{n})$ is the channel impulse response, which is equal to

$$
\mathrm{H}(\mathrm{n})=\sum_{\mathrm{i}=0}^{\mathrm{r}-1} \mathrm{~h}_{\mathrm{i}} \mathrm{e}^{\frac{\mathrm{j} 2 \pi \mathrm{f}_{\mathrm{D}} \mathrm{Tn}}{\mathrm{N}}} \delta(\lambda-\tau) \quad 0<n<N-1
$$

where $r$ is the total number of propagation paths, $h_{i}$ is the complex impulse response of the $i^{\text {th }}$ path, $f_{D i}$ is the $\mathrm{i}^{\text {th }}$ path Doppler frequency shift, $\lambda$ is delay spread index, $\mathrm{T}$ is the sample period and $\tau_{\mathrm{i}}$ is the $\mathrm{i}^{\text {th }}$ path delay normalized by the sampling time.

At the receiver, after passing to discrete domain through $\mathrm{A} / \mathrm{D}$ and low pass filter, guard time is removed:

$$
y(n)=y_{f}\left(n+N_{g}\right) \quad n=1,2, \ldots . . N-1
$$

Then $y(n)$ is sent to DFT block for the following operation

Assuming there is no ISI, then

$$
\mathrm{Y}(\mathrm{k})+\mathrm{H}(\mathrm{k})=\operatorname{DFT}\{\mathrm{h}(\mathrm{n})\}
$$

$\mathrm{I}(\mathrm{k})$ that is ICI because of Doppler frequency and $\mathrm{W}(\mathrm{k})=\operatorname{DFT}\{\mathrm{w}(\mathrm{n})\}$, with the following equation: 


\section{CHANNEL ESTIMATION IN OFDM}

(7)

Depending on the arrangement of pilots, two different types of pilot structures are considered: block type and comb type.

\subsection{Block Type}

In this type, OFDM symbols with pilots at all subcarriers are transmitted periodically for channel estimation. Channel can be estimated at pilot frequencies by two ways:

\subsection{1 (LS) Estimation}

\subsection{2 (LMMSE) Estimation}

\subsubsection{Least Square Estimation}

The idea behind least squares is to fit a model to measurements in such a way that weighted errors between the measurements and the model are minimized [4]. The LS estimate of the attenuations h, given the received data $\mathrm{Y}$ and the transmitted symbols $\mathrm{X}$ is

$$
\mathrm{h}_{\mathrm{ls}}=\mathrm{X}^{-1} \mathrm{Y}=\left[\begin{array}{lll}
\frac{\mathrm{y}_{0}}{\mathrm{x}_{0}} & \frac{\mathrm{y}_{1}}{\mathrm{x}_{1}} \ldots \ldots & \frac{\mathrm{y}_{\mathrm{N}-1}}{\mathrm{x}_{\mathrm{N}-1}}
\end{array}\right]^{\mathrm{T}}:
$$

\subsubsection{Linear Minimum Mean Square Error Estimation}

The linear minimum mean square error (LMMSE) estimate has better than the LS estimate for channel estimation in OFDM systems based on block type pilot arrangement. The LMMSE estimate has about $0-5 \mathrm{~dB}$ gain in SNR over LS estimate for the same MSE values [2]. The major drawback of the LMMSE estimate is its high complexity, which grows exponentially with observation samples [10]. In a low rank approximation is applied to a linear minimum mean squared error estimator (LMMSE estimator) that uses the frequency correlations of the channel. Assume that all the available LS estimates are arranged in a vector $\mathrm{p}$ and the channel values that have to be estimated from $\mathrm{p}$ are in a vector $\mathrm{h}$. The channel estimation problem is now to find the channel estimates $\mathrm{h}$ as a linear combination of pilot LS estimates $\mathrm{p}$. The minimum mean square error estimate for this problem is given by

$$
\mathrm{h}_{\mathrm{lmmse}}=\mathrm{R}_{\mathrm{hp}}\left(\mathrm{R}_{\mathrm{pp}}\right)^{-1} \mathrm{p}
$$

Where $R_{h p}$ is the cross-covariance matrix between $\mathrm{h}$ and the noisy pilot estimates $\mathrm{p}$, given by $\mathrm{R}_{\mathrm{hp}}=\mathrm{E}\left\{\mathrm{hp} \mathrm{H}^{\mathrm{H}}\right\}$ Where $\mathrm{R}_{\mathrm{pp}}$ is the auto-covariance matrix of the pilot estimates, and is given by

$$
\begin{gathered}
R_{p p}=E\left\{p p^{H}\right\} \\
=R_{p p}+\sigma_{n}^{2}\left(p p^{H}\right)^{-1}
\end{gathered}
$$

Where $\sigma_{\mathrm{n}}{ }^{2}$ is the variance of the additive channel noise. The superscript $(.)^{\mathrm{H}}$ denotes Hermitian transpose. Now for the case of block-type pilot channel estimation,

$$
\mathrm{h}_{\mathrm{l} \text { mmse }}=\mathrm{R}_{\mathrm{hh}}\left\{\mathrm{R}_{\mathrm{hh}}+\sigma_{\mathrm{n}}^{2}\left(\mathrm{pp}^{\mathrm{H}}\right)^{-1}\right\}^{-1} \mathrm{p}
$$

\subsection{COMB-TYPE PILOT CHANNEL ESTIMATION}

In this type, every OFDM symbol has pilot tones at the periodically-located subcarriers, which are used for a frequency-domain interpolation to estimate the channel along the frequency axis. Let $S_{f}$ be the period of pilot tones in frequency. In order to keep track of the frequency-selective channel characteristics, the pilot symbols must be placed as frequently as coherent bandwidth is. As the coherence bandwidth is determined by an inverse of the maximum delay spread $\sigma_{\max }$, the pilot symbol period must satisfy the following inequality:

$$
\mathrm{S}_{\mathrm{f}} \leq \frac{1}{\sigma_{\max }}
$$

As opposed to the block - type pilot arrangement, the comb -type pilot arrangement is suitable for fast-fading channels, but not for frequency-selective channels.

\subsubsection{Least Square estimator}

When ICI is eliminated by the GI, the received signal can be modeled with the following equation:

$$
\mathrm{Y}=\mathrm{XH}+\mathrm{W}
$$

Where $\mathrm{Y}$ is the received signal vector, $\mathrm{X}$ is a diagonal matrix of the transmitted signal,

Least square estimator of OFDM signal is given

$$
\mathrm{H}_{\mathrm{ls}}=\left(\mathrm{X}^{\mathrm{H}} \mathrm{X}\right)^{-1} \mathrm{X}^{\mathrm{H}} \mathrm{Y}
$$

Since $\mathrm{X}$ is the diagonal matrix, the estimate is reduced to

$$
\mathrm{H}_{\mathrm{LS}}=\mathrm{X}^{-1} \mathrm{Y}
$$


This indicates that the LS Estimates of the frequency response channel is simply the division of the received signal by the transmitted signal. In comb-type OFDM channel estimation, the pilot sub-channel is first identified by using the transmitted pilot channel $X_{p}$ and received pilots $Y_{p}$. The estimate of the channel at pilot sub-carriers is given by

$$
\begin{aligned}
& H_{p}(m)=\frac{Y_{p}(m)}{X_{p}(m)} \\
& m=0,1,2 \ldots ., N-1
\end{aligned}
$$

\subsubsection{Least Mean Square Estimator}

The LMS estimator uses a one-tap LMS adaptive filter at each pilot frequency. The first value is found directly through LS and the following values are calculated based on the previous estimation and the current channel output

as shown in figure. $\mathrm{e}(\mathrm{m})$ is the error signal which is formed by taking the difference between the received pilot symbol $Y_{p}(m)$ and transmitted pilot symbol $X_{p}(m)$.

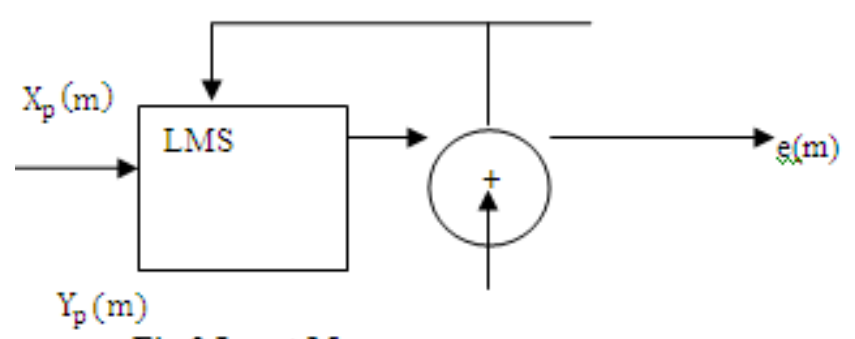

Fig.2 Least Mean square

After pilot-symbol estimation, the data on the other sub-channels is estimated by taking interpolation between the pilot sub-channel estimates.

\subsection{Block Type Estimation}

\section{SIMULATION RESULTS}

I have used a MATLAB 7.0 for simulation for the Bit Error Rate (BER) Performance of OFDM channel estimation. I simulate the BER performance of OFDM using Least square Estimation, LMMSE by using different modulation techniques like BPSK, QPSK and 16-QAM.

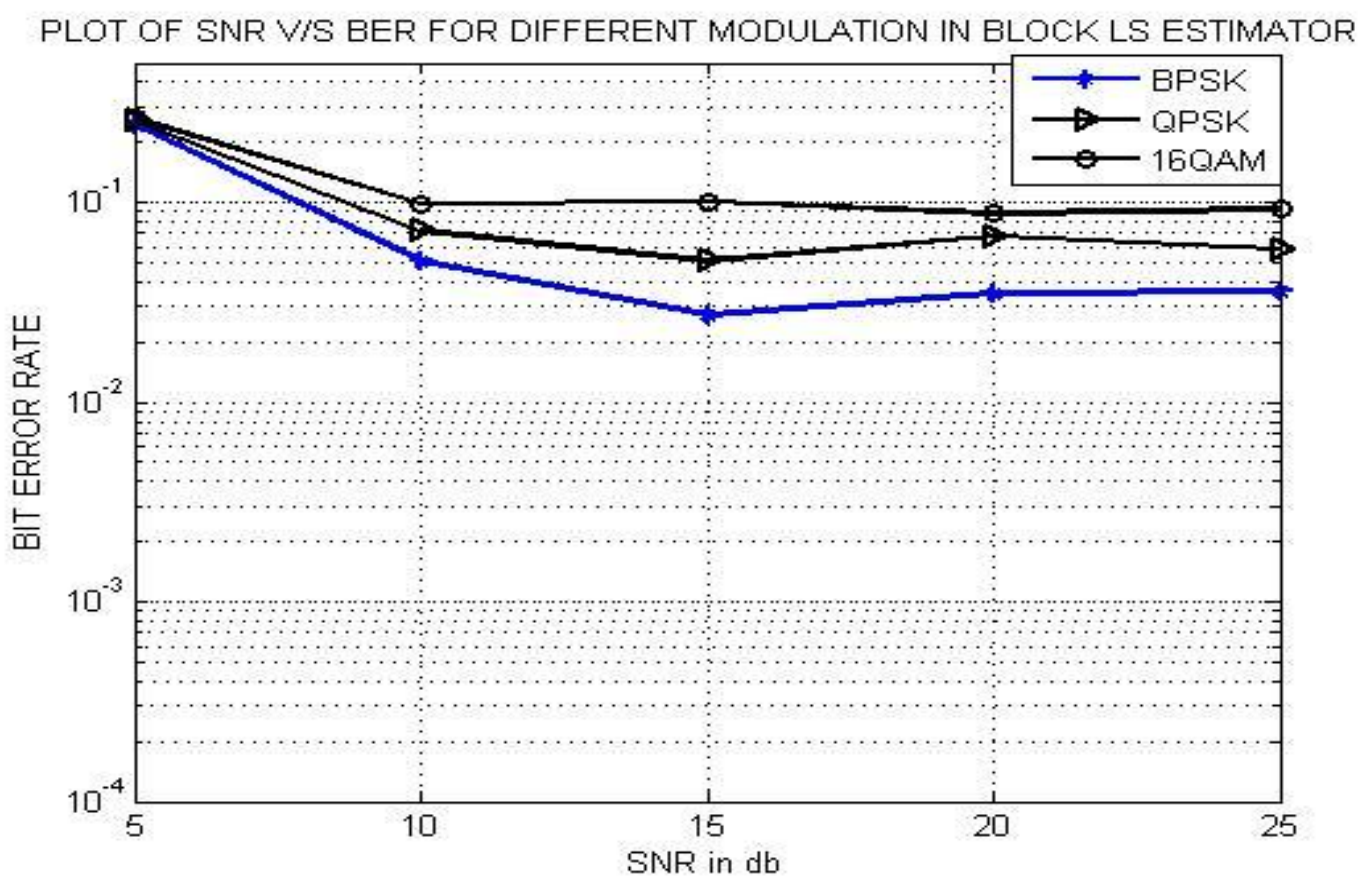

Fig.3 BER LS estimation of block Type under different modulation 


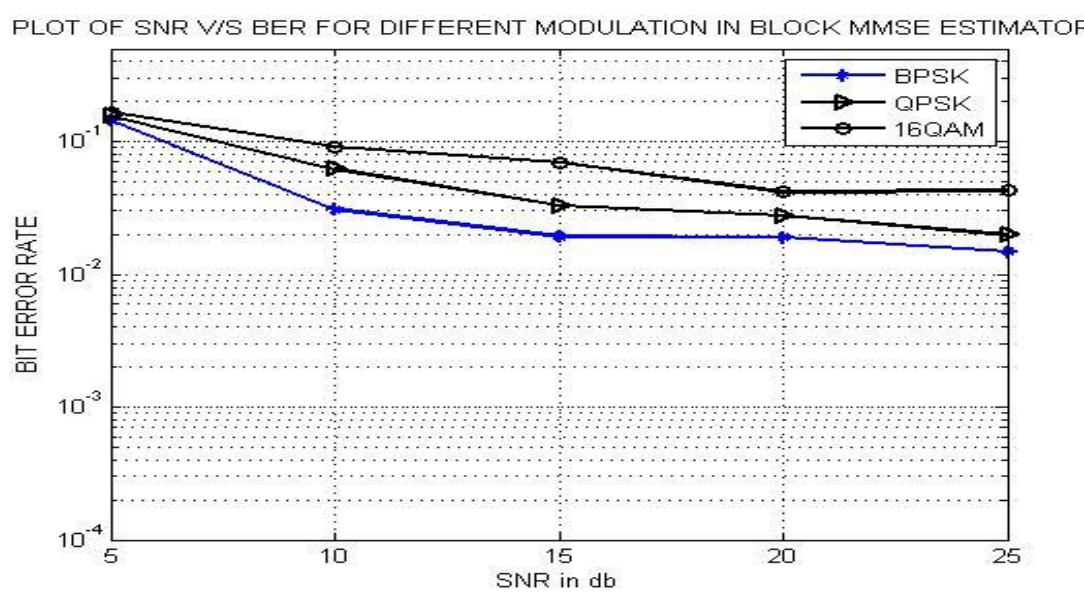

Fig.4 BER MMSE of Block type under different modulation

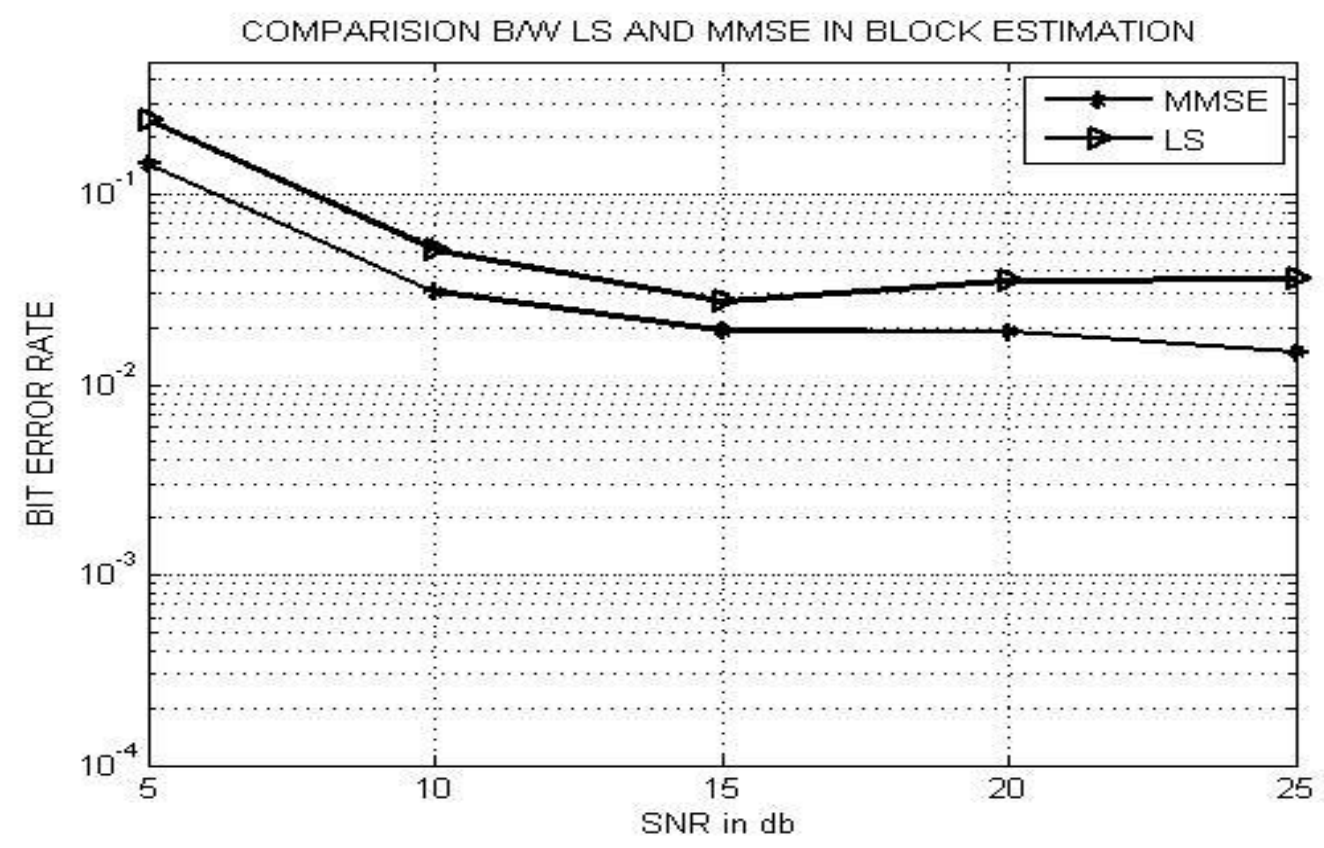

Fig.5 BER Comparison B/W LS and MMSE in block type

\subsection{Comb Type Estimation}

PLOT OF SNR VIS BER FOR DIFFERENT MODULATION IN COMB LMS ESTIMATOR

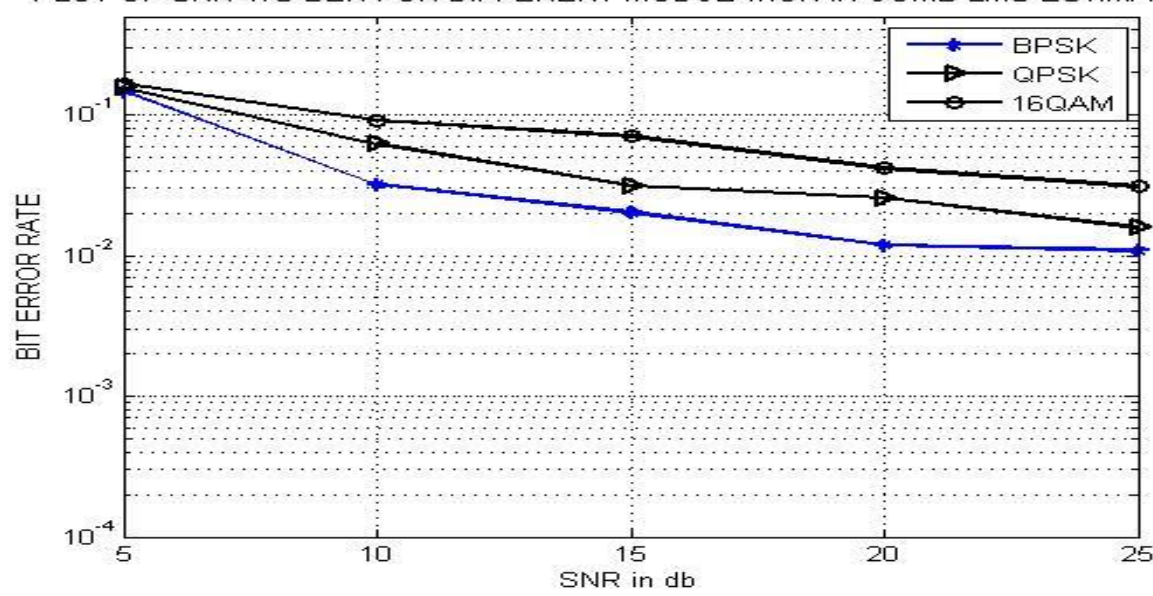

Fig.6 BER for different modulation in Comb LMS estimator 


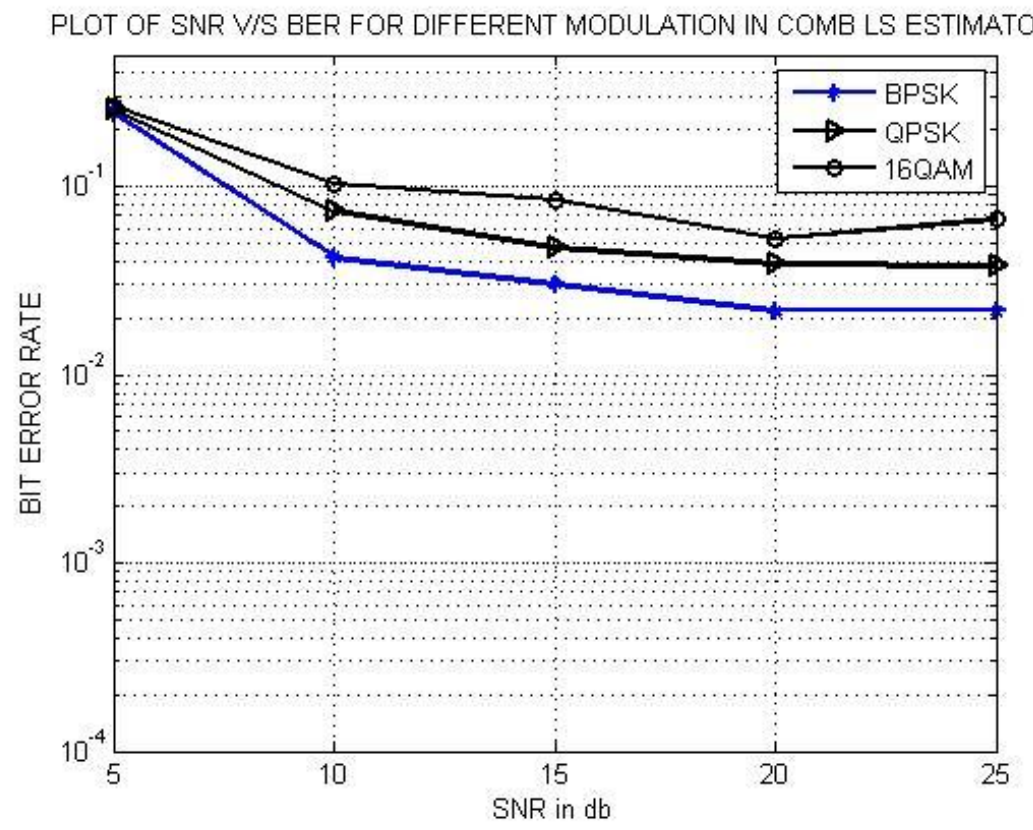

Fig.7 BER for different modulation in Comb LS Estimator

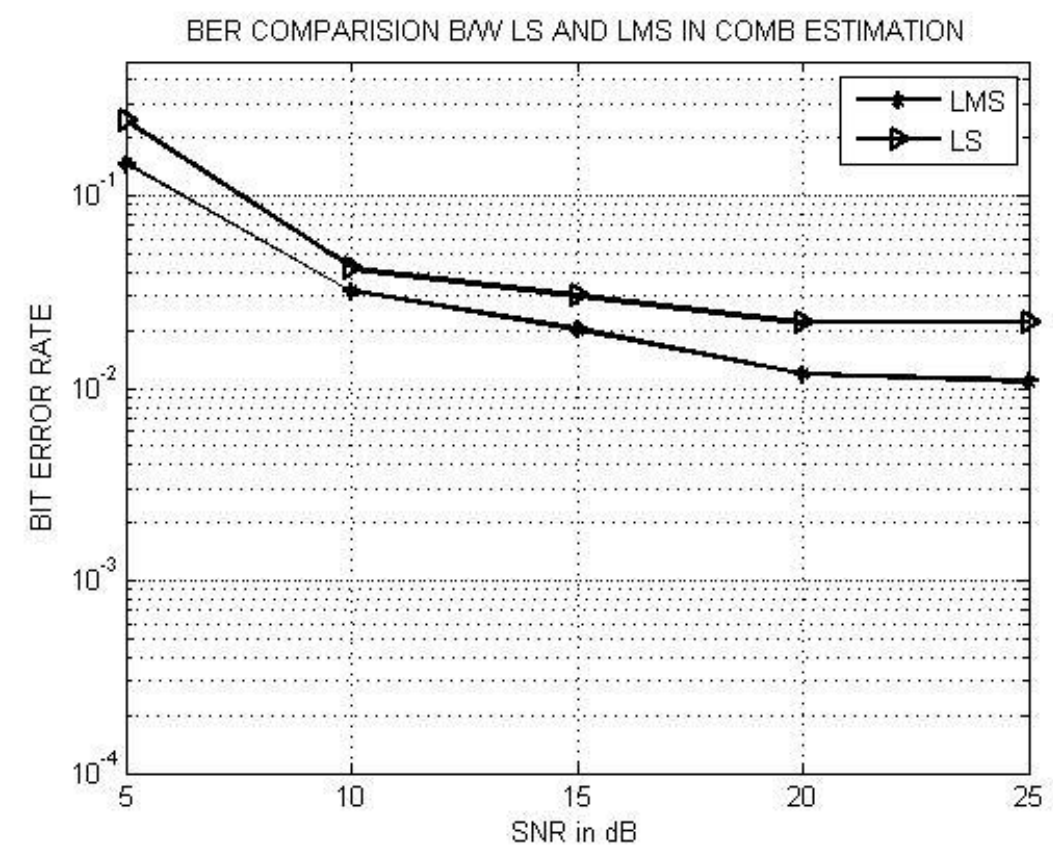

Fig.8 BER comparison B/W LS and LMS in Comb Estimation

The above graph Fig.3 Shows BER verses SNR performance of Block Type Least square estimation with BPSK, QPSK and 16QAM modulation. It is observed that the BPSK modulation has less BER as compared to the QPSK and QPSK has less BER as compared to the 16QAM.

Fig.4 shows BER verses SNR performance of Block Type MMSE with BPSK, QPSK and 16QAM modulation. It is observed that BPSK modulation has less BER as compared to the other modulation techniques. So LMMSE has good performance with BPSK.

Fig.5 shows BER verses SNR comparison between LS and LMMSE of Block Type. So it is observed that LMMSE has less Bit Error Rate as compared to the Least Square Estimation.

Fig.6 shows BER verses SNR performance of Comb type LMS Estimation with BPSK, QPSK and 16QAM.so it is observed that the BPSK modulation has less BER as compared to the QPSK and 16QAM.

Fig.7 shows BER verses SNR performance of Comb type Least square Estimation with BPSK, QPSK and 16QAM.LS estimation with BPSK has less BER as compared to the QPSK and 16QAM modulation. 
Fig. 8 shows BER verses SNR comparison of LMS and LS estimation. It is observed that LMS has less bit Error rate as compared to the Least Square Estimation.

\section{CONCLUSION}

In wideband mobile channels, Pilot based signal correction schemes has been proven a feasible method for OFDM systems. Channel estimation can be performed by many ways: either inserting pilot tones into all of the subcarriers of OFDM symbols with a specific period or inserting pilot tones into each OFDM symbol. Channel estimation based on Comb type pilot arrangement is presented, and it is shown that this technique has less Bit Error Rate as compared to the block type channel estimation. As shown in fig.8 Least Mean square has better performance as compared to the LS estimation. One of the major advantages of OFDM systems is its robustness against multipath delay spread of the channel. Hence, its typical applications are in tough radio environments. From the simulation results it is clear that if the length of guard interval is chosen properly, then OFDM systems exhibits robustness against multipath propagation eliminates ISI and hence can be used for transmission at higher data rates. OFDM is also suitable for single frequency networks, since the signals from other transmitters can also be viewed as echoes i.e. multipath propagation.

\section{REFERENCES}

[1]. M. Hsieh and C. Wei, "Channel estimation for OFDM systems based on comb-type pilot arrangement in frequency selective fading channels" in IEEE Transactions on Consumer Electronics, vol. 44, no.1, February 2002

[2]. D. J. Young and C. Beaulieu, "The generation of correlated Rayleigh random variates by inverse discrete Fourier transform," IEEE Trans on Communications, Vol. 48, No. 7, July 2000.

[3]. Y.Li, "Pilot-Symbol-Aided Channel Estimation for OFDM in Wireless Systems", in IEEE Transactions on Vehicular Technology, vol. 49, no.4, July 2000.

[4]. S. Coleri, M. Ergen, A. Puri and A. Bahai, "Channel estimation techniques based on pilot arrangement in OFDM systems," IEEE Trans. On Broadcasting, Vol. 48, No. 3, September 2002.

[5]. Yong Soo Cho, Won young Yang, "MIMO-OFDM Wireless communication With Matlab".

[6]. Uma Shanker Jha, "OFDM Towards fixed and Mobile broadband wireless Access".

[7]. T.S.Rappaport, "Wireless Communication", Prentice Hall, 2009.

[8]. R. Prasad, “OFDM for Wireless Communications Systems,” Artech House, Boston- London, 2004.

[9]. J. Heiskala, J. Terry, "OFDM Wireless LANs: A Theoretical and Practical Guide," Sams Publishing, 2002.

[10]. C. Nerguizian, M. Djadel, C. Despins, and S. Affes, "Narrowband and Wideband Radio

[11]. Channel Characteristics in Underground Mining Environments at $2.4 \mathrm{GHz}$, " Proc. of IEEE PIMRC'03, Beijing, China, Vol. 1, pp. 680-684, September 7-10, 2003.

[12]. A. Benzakour, S. Affes, C. Despins, and P.-M. Tardif, "Wideband Measurements of Channel Characteristics at 2,4 and $5.8 \mathrm{GHz}$ in Underground Mining Environments," Proc.of IEEE VTC'04-Fall, Los Angeles, California, USA, Vol. 5, pp. 3595-3599, September 26-29, 2004.

\section{BIOGRAPHY}



Mr. Vivek Kanwar received an M.Tech and B.Tech degree in Electronic and Communication Engineering from Jaypee university of information technology, solan (H.P). Now he is currently working as a Assistant Professor in Shoolini university solan, (H.P). His current research interest in the area of OFDM, MIMO, Wireless mobile communication Engineering and $4 \mathrm{G}$ wireless communications. He is the member of many international journals.

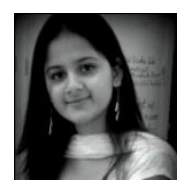

Ms. Deepika Sharma is resident of Jammu and Kashmir. She is pursuing B.Tech. in Electronics and communication Engineering from Shoolini university Solan (H.P). Her interest research in the field of wireless communication, data communication, OFDM Technology and wireless 4G Communication.

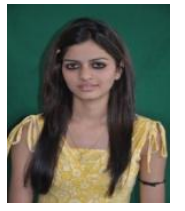

Ms. Himani Thakur is pursuing B.Tech. in Electronics and communication Engineering from Shoolini university Solan (H.P). Her interest research in the field of digital communication, data communication, MIMO Technology and wireless 3G Communication. 